\title{
Single-atom vacancy in monolayer phosphorene: a comprehensive study of stability and magnetism under applied strain
}

\author{
Juliana M. Morbec, ${ }^{1, *}$ Gul Rahman, ${ }^{1,2}$ and Peter Kratzer ${ }^{1,3}$ \\ ${ }^{1}$ Faculty of Physics, University of Duisburg-Essen, Lotharstrasse 1, 47057 Duisburg, Germany \\ ${ }^{2}$ Department of Physics, Quaid-i-Azam University, Islamabad 45320, Pakistan \\ ${ }^{3}$ Center for Nanointegration (CENIDE), University of Duisburg-Essen, Lotharstrasse 1, 47057 Duisburg, Germany
}

\begin{abstract}
Using first-principles calculations based on density-functional theory we systematically investigate the effect of applied strain on the stability and on the electronic and magnetic properties of monolayer phosphorene with single-atom vacancy. We consider two types of single vacancies: the symmetric SV-55|66, which has a metallic and non-magnetic ground state, and the asymmetric SV-5|9, which is energetically more favorable and exhibits a semiconducting and magnetic character. Our results show that compressive strain up to $10 \%$, both biaxial and uniaxial along the zigzag direction, reduces the formation energy of both single-atom vacancies with respect to the pristine configuration and can stabilize these defects in phosphorene. We found that the magnetic moment of the SV-5|9 system is robust under uniaxial strain in the range of -10 to $+10 \%$, and it is only destroyed under biaxial compressive strain larger than $8 \%$, when the system also suffers a semiconductor-to-metal transition. Additionally, we found that magnetism can be induced in the SV-55|66 system under uniaxial compressive strain larger than $4 \%$ along the zigzag direction and under biaxial tensile strain larger than $6 \%$. Our findings of small formation energies and non-zero magnetic moments for both SV-5|9 and SV-55|66 systems under zigzag uniaxial compressive strain larger than $4 \%$ strongly suggest that a magnetic configuration in monolayer phosphorene can be easily realized by single-vacancy formation under uniaxial compressive strain.
\end{abstract}

\section{INTRODUCTION}

Two-dimensional (2D) materials have been attracting increasing interest and new materials have been explored - silicene, germanene, hexagonal boron nitride, transition metal dichalcogenides, to cite a few [1-4]since the discovery of graphene in 2004 [5]. Moreover, $2 \mathrm{D}$ materials continue to be one of the most interesting topics in condensed-matter physics and materials science due to their distinctive properties and promising applications in nanoelectronics and spintronics.

In 2014, a few-layer phosphorene (the 2D counterpart of layered black phosphorus) was successfully fabricated via mechanical exfoliation of bulk black phosphorus $[6,7]$ and immediately attracted considerable attention [8-15]. Phosphorene has a characteristic puckered structure [see Fig. 1], which leads to a substantial anisotropy of its mechanical behavior [16], electric conductance [6,9] and optical responses [11], distinguishing it from many other isotropic 2D materials. It has been considered to be an attractive material for nanoelectronic applications due to its high hole mobility: values as high as $984 \mathrm{~cm}^{2} \mathrm{~V}^{-1} \mathrm{~s}^{-1}$ were obtained in few-layer phosphorene field-effect transistor with thickness of $\sim 10 \mathrm{~nm}$ [7], and a theoretical study has estimated that hole mobility along the zigzag direction may reach values in the range of $10,000-26,000 \mathrm{~cm}^{2} \mathrm{~V}^{-1} \mathrm{~s}^{-1}$ for monolayer and 3,000$4,600 \mathrm{~cm}^{2} \mathrm{~V}^{-1} \mathrm{~s}^{-1}$ for five-layer system [9]. Additionally, phosphorene has been observed to exhibit a direct band gap, with optical gaps varying from $1.73 \mathrm{eV}$ for monolayer to $0.83 \mathrm{eV}$ for the trilayer system [17], which makes

\footnotetext{
*jmmorbec@gmail.com
}

it interesting for optoelectronic applications since a large portion of the electromagnetic spectra can be covered by varying the number of layers.

Monolayer phosphorene has been theoretically predicted to sustain tensile strain up to $30 \%$ along the armchair direction and up to $27 \%$ along the zigzag direction [18]; this superior flexibility is attributed to the phosphorene puckered structure and opens an avenue for exploring strain-engineering in this system. For example, first-principles studies have found that the band gap of monolayer phosphorene can be tuned from direct to indirect with compressive strain up to $12 \%[19,20]$ and the prefered mobility direction rotates by $90^{\circ}$ under biaxial tensile strain between 3 and $6 \%$ [21]. We would like to mention that controllable strain can be applied to $2 \mathrm{D}$ materials by (i) bending a flexible substrate: after a $2 \mathrm{D}$ material has been deposited and adheres to the planar substrate, it is exposed to uniaxial tensile/compressive strain on the convex/concave side of the bent substrate, and by (ii) piezoelectric stretching: piezoelectric materials can be used to apply biaxial strain to $2 \mathrm{D}$ materials [22].

Moreover, strain-engineering in defective phosphorene has also attracted significant interest [23-25] since theoretical studies reported that single- and double-atom vacancies have lower formation energies in monolayer phosphorene when compared with graphene and silicene [26], and the asymmetric single-atom vacancy (SV-5|9), which is the most stable single-vacancy configuration in phosphorene [26, 27], may exhibit non-zero magnetic moment whereas the symmetric single-vacancy (SV-55|66) and the double-atom vacancies have non-magnetic ground state [26-29]. In this paper, we present a comprehensive investigation of the stability and the electronic and mag- 
netic properties of monolayer phosphorene with singleatom vacancy (both SV-5|9 and SV-55|66) under biaxial strain and uniaxial strain along the zigzag and armchair directions, considering both compressive and tensile strain. Using first-principles calculations, we show that both types of single vacancies can be stabilized through compressive strain, with a substantial reduction in the defect formation energy under strain up to $-10 \%$; our results also reveal that the $\mathrm{SV}-5 \mid 9$ system keeps its magnetic character under uniaxial strain in the range of -10 to $+10 \%$, but becomes non-magnetic under biaxial compressive strain larger than $8 \%$, and the non-magnetic SV-55|66 becomes magnetic under uniaxial compressive strain $(>4 \%)$ along the zigzag direction and under biaxial tensile strain larger than $6 \%$. We also observe changes in the electronic structure, with a transition direct-toindirect-to-metallic in the SV-5|9 system under biaxial compressive strain.

\section{COMPUTATIONAL DETAILS}

Spin-polarized calculations were performed in the framework of the density-functional theory [30] using the Quantum ESPRESSO code [31]. We employed the generalized gradient approximation (GGA) proposed by Perdew, Burke, and Ernzerhof (PBE) [32] for the exchange-correlation functional and ultrasoft RappeRabe-Kaxiras-Joannopoulos (RRKJ) [33] pseudopotentials to describe the electron-ion interactions. The kinetic energy cutoff for the wavefunctions was set to $40 \mathrm{Ry}$. We considered a supercell with $4 \times 4$ units of monolayer phosphorene (as shown in Fig. 1) and a vacuum region of $\sim 13 \AA$ to avoid the interaction between adjacent monolayers in neighboring supercells; we create one single-atom vacancy in this supercell, which corresponds to a defect concentration of $1 / 64 \approx 1.56 \%$. After fully optimizing the geometry of the pristine system we found $a_{0}=3.302$ and $b_{0}=4.636 \AA$ for the lateral dimensions of the unit cell, in close agreement with first-principles results previously reported in the literature $(a=3.30$ and $b=4.60 \AA$ ) [34] and with the experimental lattice constants of bulk black phosphorus $(a=3.313, b=4.374$ and $c=10.473 \AA$ ) [35]. The Brillouin zone was sampled using a $6 \times 6 \times 1$ Monkhorst-Pack [36] $k$-point grid for the geometry optimization and a denser grid, $12 \times 12 \times 1$, for the calculations of the electronic structure.

We considered uniaxial strain along the zigzag and armchair directions as well as biaxial strain. Uniaxial strain along the zigzag (armchair) direction was simulated by altering the lattice constant $a(b)$, with strength defined as $\frac{a-a_{0}}{a_{0}} \times 100 \%\left(\frac{b-b_{0}}{b_{0}} \times 100 \%\right)$, where $a_{0}\left(b_{0}\right)$ is the equilibrium lattice constant; the cell was strained in one direction and allowed to fully relax along the perpendicular direction. To simulate biaxial strain, the in-plane lattice parameters $a$ and $b$ were equally strained.

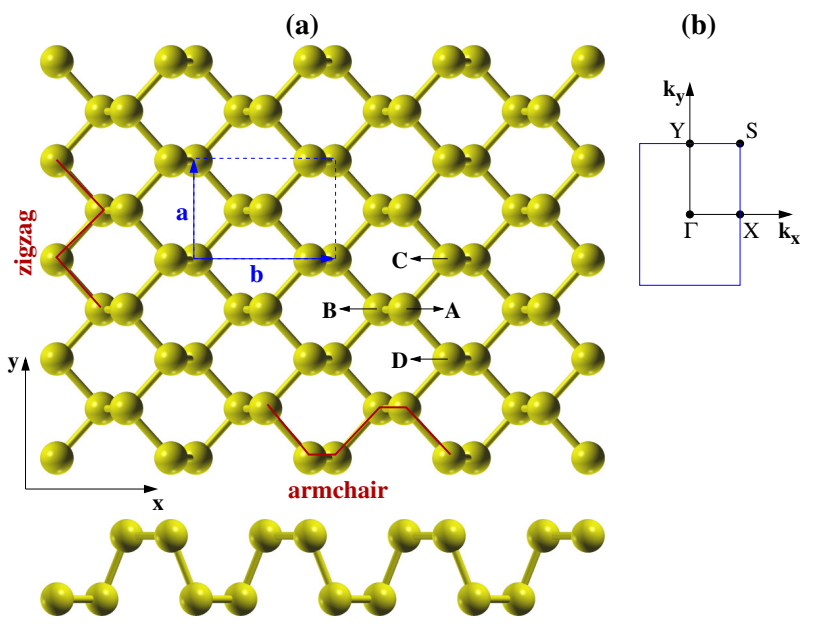

FIG. 1. (a) Ball and stick representation of monolayer phosphorene. The blue rectangle represents the unit cell ("a" and "b" are the lattice parameters) and the letters "A"- "D" indicate the key atoms for the simulation of the SV-5|9 and SV$55 \mid 66$ single-atom vacancies (see text). (b) Schematic representation of the $2 \mathrm{D}$ Brillouin zone showing the high-symmetry points.

\section{RESULTS AND DISCUSSION}

\section{A. Atomic structure and stability of single-atom vacancies}

We first investigate the atomic structure and stability of monolayer phosphorene with single-atom vacancies, considering two configurations, SV-55|66 and SV-5|9, as suggested in Ref. [26]. Both defects are created by removing the $\mathrm{P}$ atom labeled as "A" in Fig. 1. In the SV$55 \mid 66$ configuration, the vacancy reconstructs symmetrically with a four-fold coordinated atom in the center [see Fig. 2(a)]. This central atom reaches its position simply by geometry relaxation starting from the ideal lattice positions. It results in formation of two five-membered rings and two six-membered rings. In the SV-5 $\mid 9$ configuration the atom labeled as "B" was slightly moved away from its original position, and after geometry optimization two of the three dangling bonds of the $\mathrm{P}$ atoms next to the vacancy became saturated [see Fig. 2(b)], leading to the formation of a five- and a nine-membered ring, while the dangling bonds of atoms "C" and "D" remain unsaturated. Due to this partially occupied dangling bond state, the $\mathrm{SV}-5 \mid 9$ reconstruction can be stabilized by formation of a magnetic moment, while SV-55|66 has a non-magnetic ground state.

We found that $\mathrm{SV}-5 \mid 9$ is energetically more favorable than SV-55|66, which is consistent with previous theoretical studies [26, 27]; we obtained formation energies $E_{f}$ of $1.634 \mathrm{eV}$ for SV-5|9 and $2.028 \mathrm{eV}$ for SV-55|66, in excellent agreement with the values 1.626 and $2.025 \mathrm{eV}$ reported in Ref. [26]. The formation energy was com- 

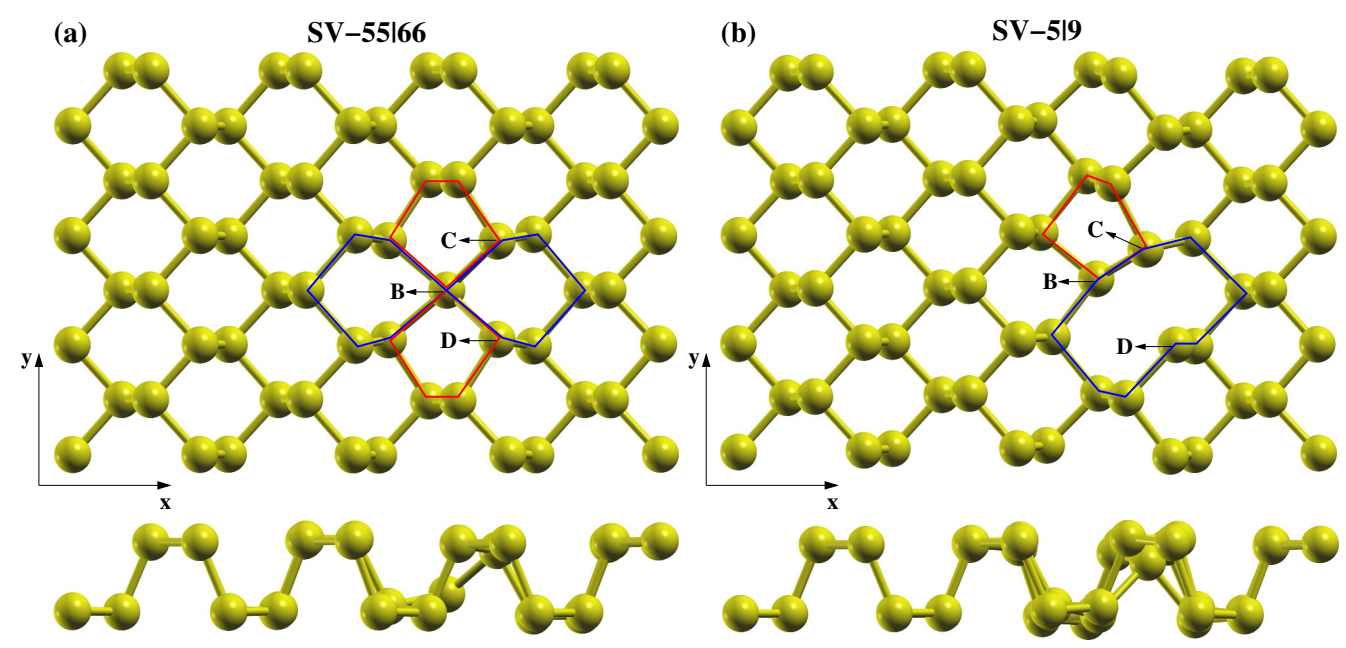

FIG. 2. Optimized geometries of monolayer phosphorene with (a) SV-55|66 and (b) SV-5|9 single-atom vacancies. The letters "B", "C" and "D" indicate the key atoms for the simulation of the defects (see also Fig. 1). Red and blue lines in (a) indicate, respectively, the five- and six-membered rings of the SV-55|66 configuration, and in (b) indicate the five- and nine-membered rings of the SV-5|9 system.

puted using

$$
E_{f}=E_{\text {def }}-E_{\text {pris }}+\mu_{P}
$$

where $E_{\text {def }}$ and $E_{\text {pris }}$ are the total energies of defective and pristine phosphorene at the same strain level, respectively, and $\mu_{P}$ is the chemical potential of phosphorus (here obtained from the unstrained bulk blackphosphorus). Negative values of $E_{f}$ indicates that defective phosphorene has lower formation energy than the pristine system under the same strain condition.

We next examine how the formation energies of the vacancies are affected by applied strain. Figure 3 shows that biaxial compressive strain and uniaxial compressive strain along the zigzag direction lead to a significant reduction in the formation energy of both the SV-55|66 and SV-5|9 systems. Under biaxial compressive strain larger than $8 \%$, the single-vacancy configurations have lower formation energies than the pristine phosphorene at the same strain level; this suggests that the formation of single-atom vacancies is easier under biaxial compressive strain. We believe that this is due to the fact that the presence of single vacancies favors the appearance of ripples, which helps to accomodate the compressive biaxial strain (we will discuss about it in more detail in the next two paragraphs). Under uniaxial compressive strain along the zigzag direction, the formation energies of SV-55|66 and SV-5|9 are reduced by more than $1.5 \mathrm{eV}$ when the applied strain varies from $0 \%$ to $-10 \%$ : $E_{f}$ of SV-55|66 is reduced from $2.028 \mathrm{eV}$ (unstrained) to $0.084 \mathrm{eV}$ (strain of $-10 \%$ ), and $E_{f}$ of SV-5|9 is reduced from $1.634 \mathrm{eV}$ (at $0 \%$ ) to $0.114 \mathrm{eV}$ (at $-10 \%$ ). We notice, on the other hand, that uniaxial compressive strain along the armchair direction has a weaker effect on the formation energies of the vacancies: $E_{f}$ of SV-55|66 and $\mathrm{SV}-5 \mid 9$ are reduced by only about 0.6 and $0.4 \mathrm{eV}$, respectively, under a compressive strain of $-10 \%$. Similarly, tensile strain (biaxial and uniaxial) is also observed to only slightly affect the formation energy of the SV-55|66 and SV-5|9 systems [see Fig. 3]: $E_{f}$ varies by less than $0.5 \mathrm{eV}$ for SV-55|66 and by less than $0.3 \mathrm{eV}$ for SV-5|9 under applied strain in the range of 0 to $+10 \%$.

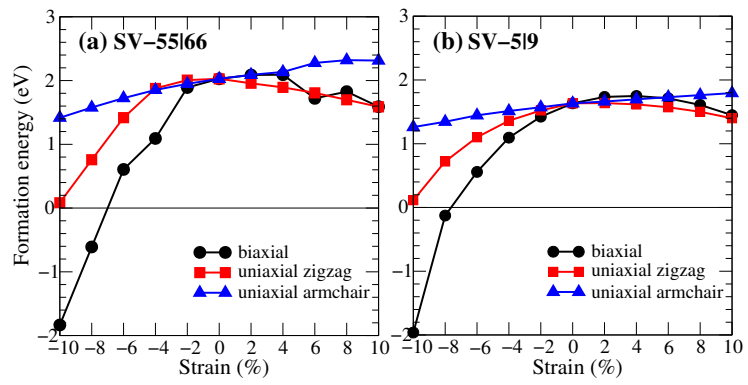

FIG. 3. Formation energy of the (a) SV-55|66 and (b) SV$5 \mid 9$ systems under applied strain. Black circles, red squares and blue triangles correspond to biaxial, uniaxial-zigzag and uniaxial-armchair strain, respectively.

Our results clearly show that compressive strain, especially biaxial strain and uniaxial strain along the zigzag direction, can stabilize single-atom vacancies in monolayer phosphorene, by significantly reducing their formation energies. We found that the stability of the defects under biaxial compressive strain is associated with the appearance of a ripple deformation, as shown in Fig. 4. In the SV-55|66 configuration [Fig. 4(a)], the ripple starts at $-4 \%$ strain and becomes more pronounced at strain beyond $-8 \%$; we found that the difference between the heights of the highest and the lowest $\mathrm{P}$ atoms in the monolayer increases from $2.30 \AA$ in the unstrained system to $4.37 \AA$ at $-8 \%$ and $5.26 \AA$ at $-10 \%$. In the SV-5|9 system [Fig. 4(b)] the deformation is small for strain up to $-8 \%$ and increases substantially at $-10 \%$, with the dif- 
ference between the heights of the highest and the lowest $\mathrm{P}$ atoms increasing from $2.52 \AA$ at $0 \%$ to $5.63 \AA$ at $-10 \%$.

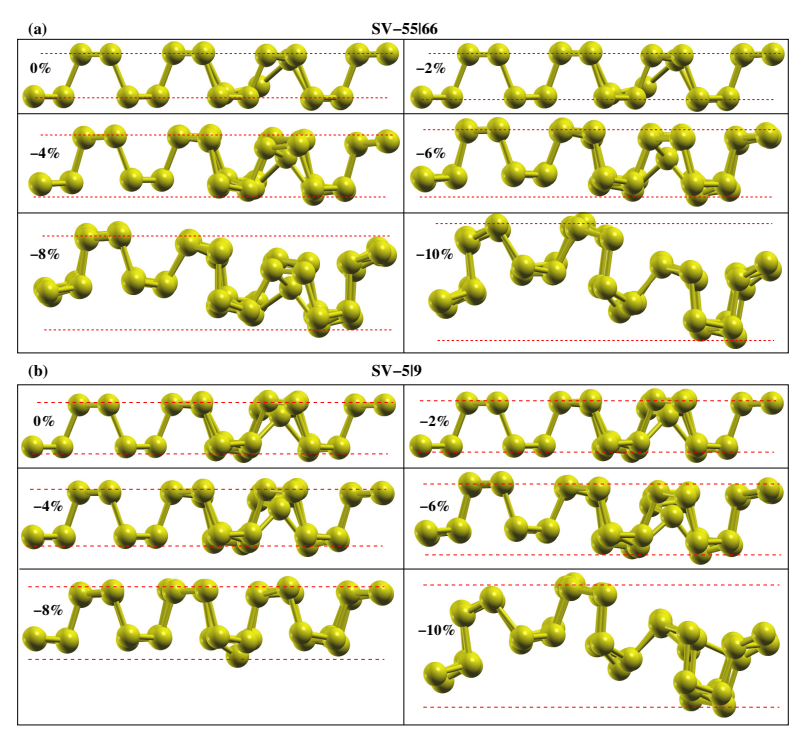

FIG. 4. Side view of the optimized geometries of the (a) SV$55 \mid 66$ and (b) SV-5|9 systems under biaxial compressive strain. The dashed red lines are guidelines for the highest and lowest $\mathrm{P}$ atoms in the monolayer.

Compression-induced ripple deformation in pristine monolayer phosphorene has been reported in a previous theoretical study by Kou et al. [37]; they found that the deformation is highly anisotropic, occurring mainly along the zigzag direction at compressive strain up to $10 \%$, while only a small out-of-plane deformation was observed to occur along the armchair direction. In the present work, we did not observe the formation of ripples in pristine phosphorene under compressive strain up to $10 \%$, possibily because we used a small supercell (here we use a supercell with 4 unit cells while Kou et al. [37] used at least 11 unit cells to simulate the ripple deformation). However, we did observe ripple deformation in defective systems [see Fig. 4], even using a small supercell, which indicates that the presence of vacancies favors the formation of short-range ripples, whereas in the pristine system the deformation seems to have a larger extension. It is worth mentioning that we observed short-range ripples in the SV-55|66 and SV-5|9 systems only under biaxial strain; uniaxial compressive strain up to $10 \%$ along either the armchair or the zigzag direction does not induce ripple deformation in the small supercell we simulated in this work [see Figs. S1 and S2 in the Supplemental Material]. We believe that this is due to the fact that uniaxial compressive strain applied along one direction leads to a tensile strain along the perpendicular in-plane direction, which releases part of the strain energy and prevents the formation of short-range ripples.

\section{B. Robustness of magnetic moment in the SV-5|9 single vacancy under strain}

After investigating the stability of single-atom vacancies in monolayer phosphorene, we turn to the study of the magnetic and electronic properties of these systems. We start with the SV-5|9 single vacancy, which has a semiconducting and magnetic ground state in the unstrained regime. We found that the $\mathrm{SV}-5 \mid 9$ system has a direct band gap $(\Gamma-\Gamma)$ of about $1.06 \mathrm{eV}$ for the spin-up channel and an indirect band gap $(\mathrm{Y}-\Gamma)$ of approximately the same value for the spin-down channel [see Fig. 5(b)]. These values, which are in good agreement with previous theoretical studies [25-27], are slightly larger than the band gap calculated for the pristine monolayer, $\sim 0.93 \mathrm{eV}$ ( $\Gamma-\Gamma$ direct gap) [Fig. 5(a)]. We also found that the unstrained SV-5 $\mid 9$ system has a total magnetic moment of $\sim 1.01 \mu_{B}$ /cell (in excellent agreement with Refs. 25-27), mainly coming from the unsaturated dangling bonds of the $\mathrm{P}$ atoms around the vacancy, as can be seen from the spin-density difference depicted in Figs. 6(f) and (g). The largest magnetic moments are located in the atoms 2, 3 and 4 of Fig. 6(f): namely, $\sim 0.06 \mu_{B}$ in the atom 2 , coming from an unoccupied $p_{x}$ orbital [see Fig. 6(c)], and $\sim 0.58$ and $0.07 \mu_{B}$ in the atoms 3 and 4 , respectively, mainly from a mixture of unoccupied $p_{y}$ and $p_{z}$ states [Fig. 6(d) and (e)].

The band structure displayed in Fig. 5(b) shows a localized unoccupied state in the spin down channel of the SV-5|9 system, originated predominantly from the unsaturated dangling bonds around the vacancy; no spinpolarization is observed in the conduction band, but large spin-polarization near the Fermi energy and small polarization in the valence band can be seen. We notice that the exchange splitting at the $\Gamma$ point in the valence band (just below the Fermi level) is small, but it increases when electrons move in the X-S direction in the Brillouin Zone of phosphorene, and about $0.2 \mathrm{eV}$ exchange splitting at the $\mathrm{X}$ point can be seen. Therefore, the exchange energy increases in the armchair $(\Gamma-\mathrm{X})$ direction and decreases in the zigzag $(\Gamma-Y)$ direction; different values of exchange splitting at different points of the Brillouin Zone reflect the structural anisotropy of phosphorene.

Next, we examine the robustness of the magnetic moment and the changes in the band gap of the SV-5|9 system under applied strain. From the above analysis it is clear that the SV-5|9 single vacancy has the character of an electronic radical due to the dangling bond at atom 3 [Fig. 6] which induces a spin-polarized band in the band gap of phosphorene. Applying strain may shift the energetic position of this band relative to the valence and conduction bands of phosphorene, but the spin polarization of this band will persist under a wide range of strain values. As can be seen in Fig. 7(a), uniaxial strain along both the zigzag and armchair directions does not cause significant changes in the magnetic state of the SV-5|9 system, whose magnetic moment remains approximatelly $1 \mu_{B}$ /cell within the range of -10 

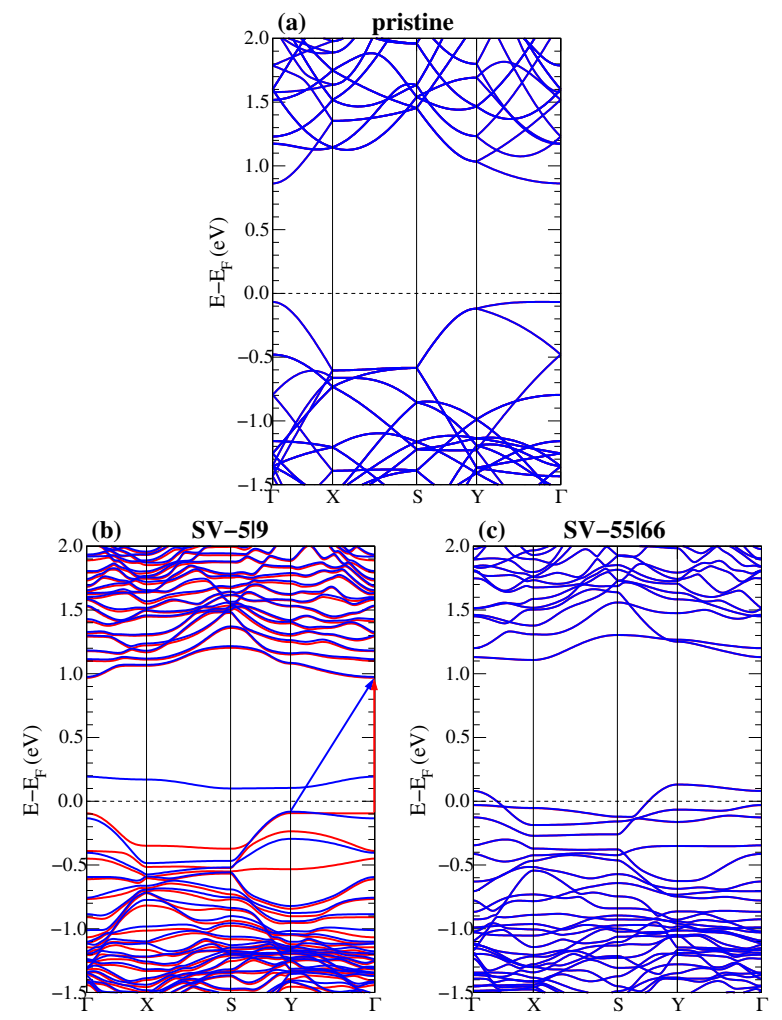

FIG. 5. Band structures of (a) pristine, (b) SV-5|9 and (c) SV$55 \mid 66$ monolayer phosphorene. Red and blue lines correspond to the spin-up and spin-down bands, respectively (spin-up and spin-down bands are coincident in (a) and (c)). In (b), red and blue arrows indicate the lowest band gap for the spinup and spin-down channels, respectively. The high-symmetry points of the Brillouin zone are shown in Fig. 1(b).

to $+10 \%$ strain. This is particularly interesting since we found that uniaxial compressive strain along the zigzag direction can stabilize the SV-5|9 single-atom vacancy by substantially reducing its formation energy with respect to the pristine system at the same strain level [see Fig. 3]. Our results therefore suggest that a magnetic configuration in monolayer phosphorene can be easier to realize under uniaxial compressive strain up to $10 \%$.

We found, on the other hand, that biaxial compressive strain larger than $6 \%$ destroys the magnetic moment of the SV-5|9 system [Fig. 7(a)], which coincides with a semiconductor-to-metal transition as shown in Figs. 7(b) and $(\mathrm{c})$; this finding is consistent with theoretical results reported in Ref. 25. We observed that large biaxial compressive strain distorts the structure in such a way that $\mathrm{P}$ atoms around the vacancy form new bonds (mostly metallic) and no dangling bonds are formed [see Fig. S4 in the Supplemental Material], which leads to no-uncompensated spins in the monolayer phosphorene. An analysis of the band structures of the SV-5|9 system under biaxial strain [see Fig. S5 in the Supplemental Material] reveals that under compression an occupied spin-up state from the valence band moves to higher energies, towards the conduction band, and, for strain be-
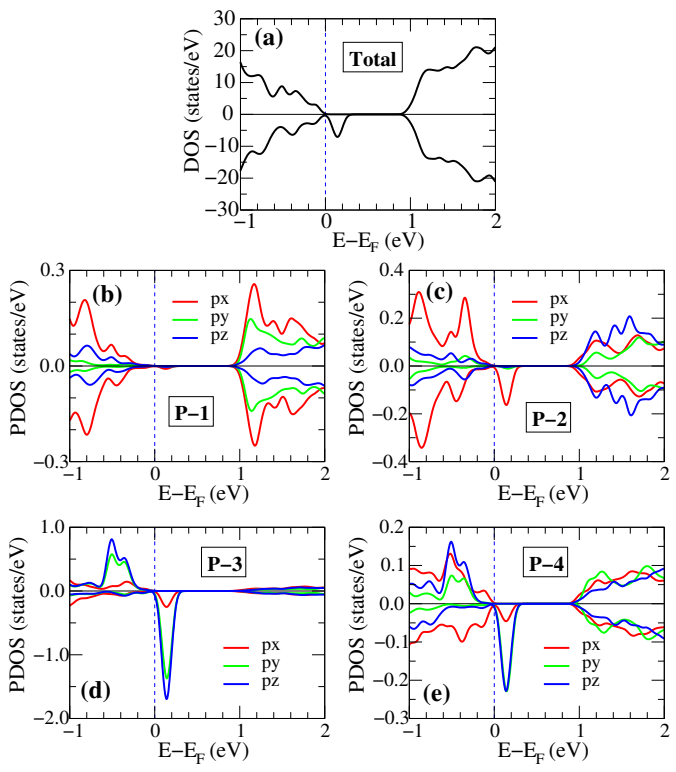

(f)

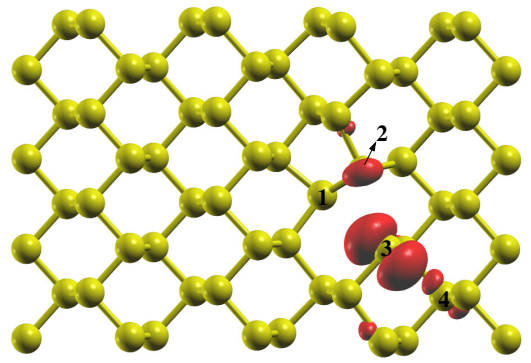

(g)

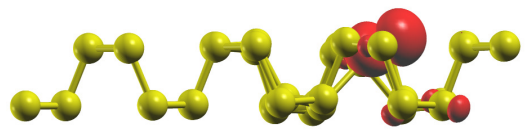

FIG. 6. (a) Total density of states (DOS), (b-e) projected density of states (PDOS) and $(\mathrm{f}+\mathrm{g})$ spin-density difference of the unstrained SV-5|9 system. The PDOS is shown for the atoms (b) 1 , (c) 2, (d) 3, and (e) 4, as indicated in (f). The spin-density difference $\Delta \rho=\rho^{\uparrow}-\rho^{\downarrow}$ is shown for an isosurface of $0.003 \mathrm{e} / \mathrm{bohr}^{3}$, where red and blue isosurfaces indicate positive and negative $\Delta \rho$, respectivelly.

yond $-6 \%$, becomes degenerated with the unoccupied spin-down state originated from the vacancy (and already present in the unstrained regime), giving rise to a state crossing the Fermi level.

Finally, we notice that the applied strain seems to have a stronger influence on the character of the band gap of the spin-up channel than that of the spin-down channel: while the spin-up bands suffer a direct-to-indirect transition under both biaxial and uniaxial strain [Fig. 7(b)], the spin-down channel preserves its indirect gap under uniaxial strain along the armchair direction and under biaxial strain in the range of -6 to $+10 \%$, and an indirect-todirect transition only occurs for uniaxial tensile strain larger than $4 \%$ along the zigzag direction [Fig. 7(c)]. It is important to mention, however, that the differences between direct and indirect band gaps are quite small in 

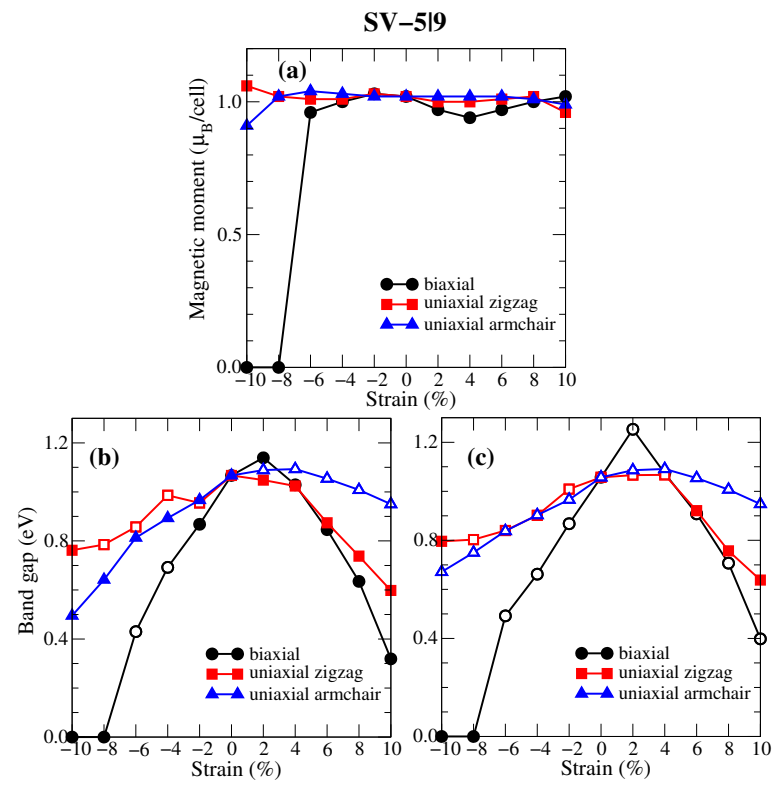

FIG. 7. (a) Magnetic moments and $(b+c)$ band gaps ((b) spin up and (c) spin down channels) of the SV-5|9 system under applied strain. Black circles, red squares and blue triangles correspond to biaxial, uniaxial-zigzag and uniaxial-armchair strain, respectively. In (b) and (c), open and closed symbols correspond to indirect and direct band gaps, respectively.

several cases due to the small dispersion of the bands; for example, as can be seen in Fig. 5(b), unstrained SV-5|9 system has nearly flat top valence and bottom conduction bands along the $\mathrm{Y}-\Gamma$ direction, which makes the values of the direct and indirect band gaps very close to each other.

\section{Strain-induced magnetic moment in the SV-55|66 single vacancy}

We now turn to the SV-55|66 single-atom vacancy which, in contrast to the SV-5|9 vacancy, was found to be non-magnetic in the unstrained regime. The deficiency of valence electrons due to the removal of one $\mathrm{P}$ atom induces hole pockets at the $\Gamma$-point visible in Fig. 5(c); however, these holes are rather delocalized, such that exchange splitting remains energetically unfavorable and no spin-split bands occurs. The SV-55|66 system was also found to be metallic [Fig. 5(c)], again in contrast to the semiconducting character of the SV-5 $\mid 9$ system. The metallic and non-magnetic character obtained here for the unstrained SV-55|66 is consistent with previous theoretical studies [23, 25, 26].

The changes in the magnetic moment and in the band gap of the SV-55|66 system are shown in Fig. 8. We found that magnetic moments between 0.9 and $1.1 \mu_{B} /$ cell are induced in the SV-55|66 system under uniaxial compressive strain larger than $4 \%$ along the zigzag direction and under biaxial tensile strain larger than $6 \%$. In these regimes we also observed a metal-to-semiconductor transition, with band gaps between 0.4 and $1.0 \mathrm{eV}$ [see Fig. 8(b) and (c)]. The emergence of magnetism in the SV-55|66 system under biaxial tensile strain has also been reported in previous theoretical studies by Chintalapati et al. [23] and by Ren at al. [25]. However, these studies did not consider uniaxial compressive strain, which in the present work has been found to be an important regime; in particular, we found that compressive strain larger than $4 \%$ along the zigzag direction can significantly reduces the formation energy of the SV-55|66 single vacancy [see Fig. 3(a)] in addition to inducing a magnetic moment of $\sim 1 \mu_{B}$ to the system [Fig. 8(a)]. This finding strongly suggests that a magnetic configuration in monolayer phosphorene can be easily realized by singlevacancy formation under uniaxial compressive strain up to $10 \%$.
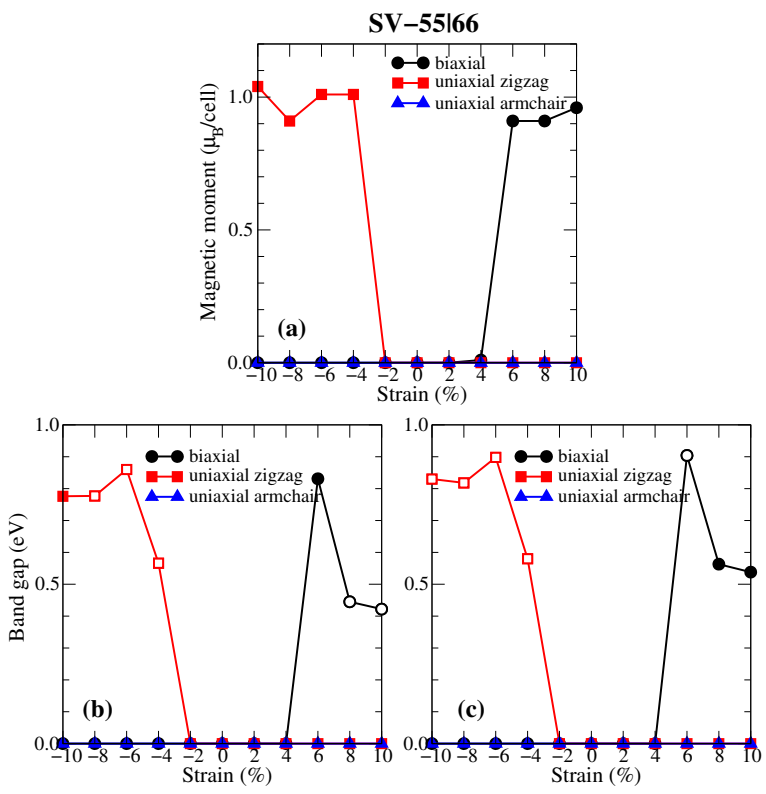

FIG. 8. (a) Magnetic moments and $(b+c)$ band gaps ((b) spin up and (c) spin down channels) of the SV-55|66 system under applied strain. Black circles, red squares and blue triangles correspond to biaxial, uniaxial-zigzag and uniaxial-armchair strain, respectively. In (b) and (c), open and closed symbols correspond to indirect and direct band gaps, respectively.

In order to gain an insight into the origin of the magnetism in the strained SV-55|66 system, we examine in detail the extreme cases where the magnetic moment develops. Figure 9 shows the density of states and the spindensity difference for the SV-55|66 system under biaxial strain of $+10 \%$. We observe that, as a result of the biaxial tensile strain, a reconstruction with broken symmetry becomes energetically more favorable than the symmetric SV-55|66 vacancy: the formerly four-fold coordinated central atom moves off-center, breaking two of its bonds, and giving rise to two dangling bonds at atoms 2 and 3 . The magnetic moment is mainly contributed by the $p_{x}$ and $p_{z}$ orbitals of these atoms [see Figs. 9(c) and (d)], 
$\sim 0.38 \mu_{B}$ at atom 2 and $0.35 \mu_{B}$ at atom 3 ; a negative spin-polarization $\left(-0.21 \mu_{B}\right)$ was found in atom 1 , mostly contributed by $p_{x}$ orbitals [Fig. $9(\mathrm{~b})$ ].
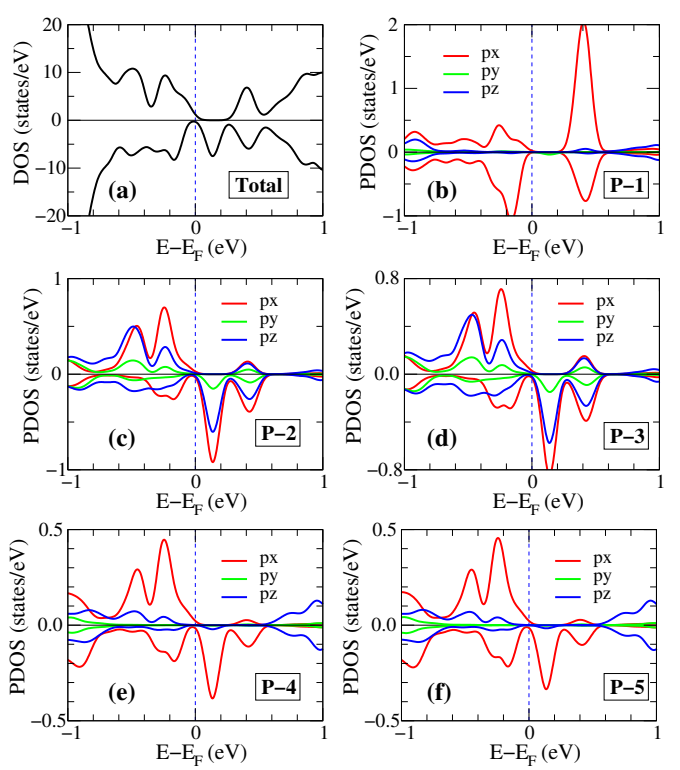

(g)

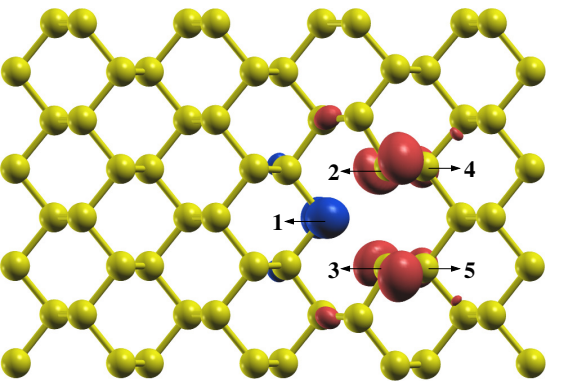

(h)

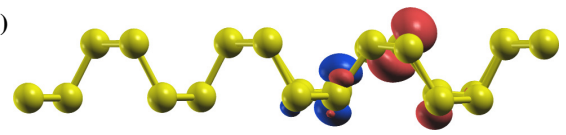

FIG. 9. (a) Total density of states (DOS), (b-f) projected density of states $(\mathrm{PDOS})$ and $(\mathrm{g}+\mathrm{h})$ spin-density difference of the SV-55|66 system under biaxial strain of $+10 \%$. The PDOS is shown for the atoms (b) 1 , (c) 2, (d) 3, (e) 4, and (f) 5 , as indicated in (g). The spin-density difference $\Delta \rho=$ $\rho^{\uparrow}-\rho^{\downarrow}$ is shown for an isosurface of $0.003 \mathrm{e} / \mathrm{bohr}^{3}$, where red and blue isosurfaces indicate positive and negative $\Delta \rho$, respectivelly.

Finally, Figure 10 shows the density of states and the spin-density difference for the SV-55|66 system under uniaxial strain of $-10 \%$ along the zigzag direction. We observe again a structural rearrangement of the atoms: due to the compression, atoms 6 and 7 approach each other and form a new bond (a new 5-membered ring is formed), while the formerly four-fold coordinated central atom 1 loses two of its bonds. The magnetic moment of the system is mainly contributed by the $p_{x}$ and $p_{z}$ orbitals of atom 1 [Fig. 10(b)], which gives about $0.47 \mu_{B}$.
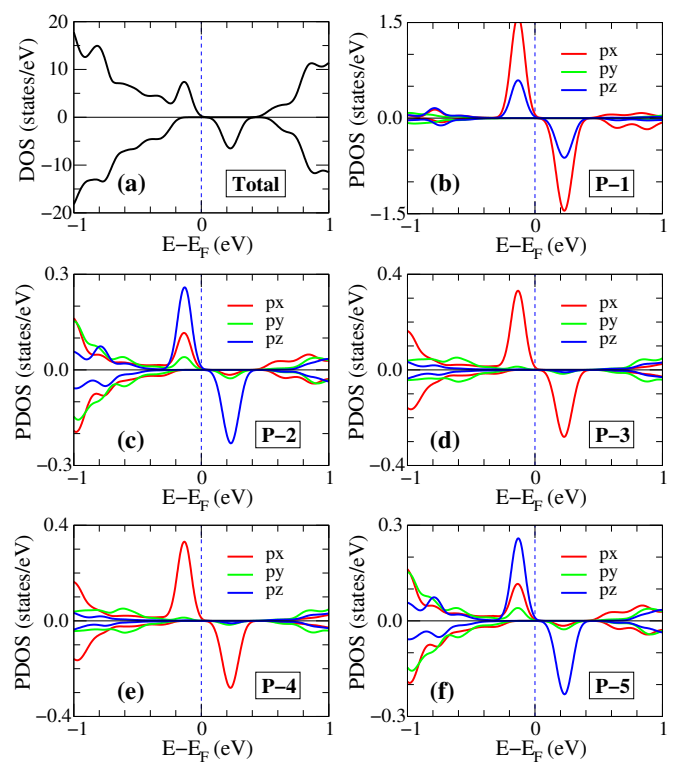

(g)

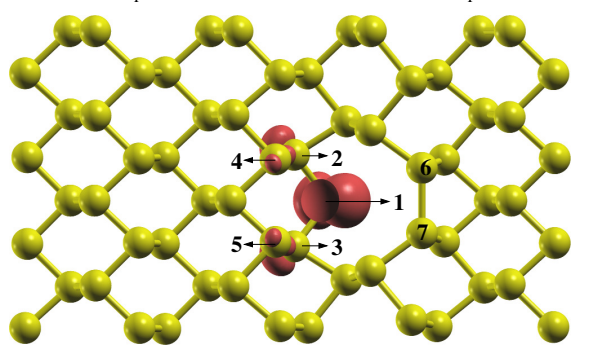

(h)

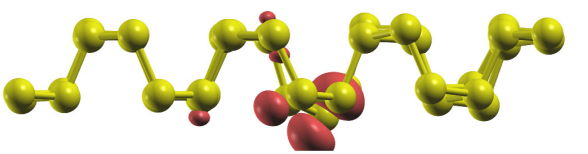

FIG. 10. (a) Total density of states (DOS), (b-f) projected density of states (PDOS) and $(\mathrm{g}+\mathrm{h})$ spin-density difference of the SV-55|66 system under uniaxial strain of $-10 \%$ along the zigzag direction. The PDOS is shown for the atoms (b) 1 , (c) 2, (d) 3, (e) 4, and (f) 5, as indicated in (g). The spindensity difference $\Delta \rho=\rho^{\uparrow}-\rho^{\downarrow}$ is shown for an isosurface of $0.003 \mathrm{e} / \mathrm{bohr}^{3}$, where red and blue isosurfaces indicate positive and negative $\Delta \rho$, respectivelly.

\section{CONCLUSIONS}

We systematically investigated the effects of applied strain on the stability and on the electronic and magnetic properties of monolayer phosphorene with singleatom vacancy. We considered two types of single vacancies: the symmetric SV-55|66, which is metallic and nonmagnetic, and the asymmetric SV-5|9, which is the most favorable single-vacancy defect in phosphorene, and has a semiconducting and magnetic ground state. Both singleatom vacancies have high formation energies $(>1.5 \mathrm{eV})$ in monolayer phosphorene, but here we show that applied strain can significantly reduce the formation energy and lead to the stabilization of these defects: in particular, we found that uniaxial compressive strain up to $10 \%$ along the zigzag direction can reduce the formation energies of 
both defects to values lower than $0.2 \mathrm{eV}$ with respect to the pristine system at the same strain condition. Interestingly, we found that both SV-5|9 and SV-55|66 single vacancies exhibit a magnetic moment of about $1 \mu_{B} /$ cell in these regimes. For the SV-5|9 system, our results show that the magnetic configuration already present in the unstrained regime is preserved under uniaxial strain in the range of -10 to $+10 \%$ along both the zigzag and armchair directions, and also under biaxial strain in the range of -6 to $+10 \%$; the magnetic state is only destroyed under biaxial compressive strain larger than $8 \%$, when the system also undergoes a semiconductor-to-metal transition. For the SV-55|66 system, which is non-magnetic and metallic in the unstrained regime, we observed a transition to a semiconducting and magnetic (with magnetic moment of $\sim 1 \mu_{B}$ /cell) configuration at uniaxial compressive strain larger than $4 \%$ along the zigzag direc- tion and for biaxial tensile strain larger than $6 \%$. Our results showing small formation energies and non-zero magnetic moments for both SV-5|9 and SV-55|66 systems under zigzag uniaxial compressive strain larger than $4 \%$ strongly suggest that a magnetic configuration in monolayer phosphorene can be easily realized by singlevacancy formation under uniaxial compressive strain.

\section{ACKNOWLEDGMENTS}

J.M.M. and P.K. gratefully acknowledge the computing time granted by the Center for Computational Sciences and Simulation (CCSS) of the University of Duisburg-Essen and provided on the supercomputer magnitUDE (DFG Grant No. INST 20876/209-1 FUGG and INST 20876/243-1 FUGG) at the Zentrum für Informations-und Mediendienste (ZIM).
[1] Hamid Oughaddou, Hanna Enriquez, Mohammed Rachid Tchalala, Handan Yildirim, Andrew J. Mayne, Azzedine Bendounan, Gérald Dujardin, Mustapha Ait Ali, and Abdelkader Kara, "Silicene, a promising new 2d material," Progress in Surface Science 90, 46-83 (2015), special Issue on Silicene.

[2] M. E. Dávila, L. Xian, S. Cahangirov, A. Rubio, and G. Le Lay, "Germanene: a novel two-dimensional germanium allotrope akin to graphene and silicene," New Journal of Physics 16, 095002 (2014).

[3] Li Song, Lijie Ci, Hao Lu, Pavel B. Sorokin, Chuanhong Jin, Jie Ni, Alexander G. Kvashnin, Dmitry G. Kvashnin, Jun Lou, Boris I. Yakobson, and Pulickel M. Ajayan, "Large scale growth and characterization of atomic hexagonal boron nitride layers," Nano Letters 10, 3209-3215 (2010).

[4] Qing Hua Wang, Kourosh Kalantar-Zadeh, Andras Kis, Jonathan N. Coleman, and Michael S. Strano, "Electronics and optoelectronics of two-dimensional transition metal dichalcogenides," Nature Nanotechnology 7, 699712 (2012).

[5] K. S. Novoselov, A. K. Geim, S. V. Morozov, D. Jiang, Y. Zhang, S. V. Dubonos, I. V. Grigorieva, and A. A. Firsov, "Electric field effect in atomically thin carbon films," Science 306, 666-669 (2004).

[6] Han Liu, Adam T. Neal, Zhen Zhu, Zhe Luo, Xianfan Xu, David Tománek, and Peide D. Ye, "Phosphorene: An unexplored 2d semiconductor with a high hole mobility," ACS Nano 8, 4033-4041 (2014).

[7] Likai Li, Yijun Yu, Guo Jun Ye, Qingqin Ge, Xuedong Ou, Hua Wu, Donglai Feng, Xian Hui Chen, and Yuanbo Zhang, "Black phosphorus field-effect transistors," Nature Nanotechnology 9, 372-377 (2014).

[8] Eugenie Samuel Reich, "Phosphorene excites materials scientists," Nature 506, 19 (2014).

[9] Jingsi Qiao, Xianghua Kong, Zhi-Xin Hu, Feng Yang, and Wei Ji, "High-mobility transport anisotropy and linear dichroism in few-layer black phosphorus," Nature Communications 5, 4475 (2014).
[10] Fengnian Xia, Han Wang, and Yichen Jia, "Rediscovering black phosphorus as an anisotropic layered material for optoelectronics and electronics," Nature Communications 5, 4458 (2014).

[11] Vy Tran, Ryan Soklaski, Yufeng Liang, and Li Yang, "Layer-controlled band gap and anisotropic excitons in few-layer black phosphorus," Phys. Rev. B 89, 235319 (2014).

[12] Michele Buscema, Dirk J. Groenendijk, Sofya I. Blanter, Gary A. Steele, Herre S. J. van der Zant, and Andres Castellanos-Gomez, "Fast and broadband photoresponse of few-layer black phosphorus field-effect transistors," Nano Letters 14, 3347-3352 (2014).

[13] A. N. Rudenko and M. I. Katsnelson, "Quasiparticle band structure and tight-binding model for single- and bilayer black phosphorus," Phys. Rev. B 89, 201408 (2014).

[14] Shengjun Yuan, A. N. Rudenko, and M. I. Katsnelson, "Transport and optical properties of single- and bilayer black phosphorus with defects," Phys. Rev. B 91, 115436 (2015).

[15] A. N. Rudenko, S. Brener, and M. I. Katsnelson, "Intrinsic charge carrier mobility in single-layer black phosphorus," Phys. Rev. Lett. 116, 246401 (2016).

[16] Luqing Wang, Alex Kutana, Xiaolong Zou, and Boris I. Yakobson, "Electro-mechanical anisotropy of phosphorene," Nanoscale 7, 9746-9751 (2015).

[17] Likai Li, Jonghwan Kim, Chenhao Jin, Guo Jun Ye, Diana Y. Qiu, Felipe H. da Jornada, Zhiwen Shi, Long Chen, Zuocheng Zhang, Fangyuan Yang, Kenji Watanabe, Takashi Taniguchi, Wencai Ren, Steven G. Louie, Xian Hui Chen, Yuanbo Zhang, and Feng Wang, "Direct observation of the layer-dependent electronic structure in phosphorene," Nature Nanotechnology 12, 21-25 (2017).

[18] Qun Wei and Xihong Peng, "Superior mechanical flexibility of phosphorene and few-layer black phosphorus," Applied Physics Letters 104, 251915 (2014).

[19] Xihong Peng, Qun Wei, and Andrew Copple, "Strainengineered direct-indirect band gap transition and its mechanism in two-dimensional phosphorene," Phys. Rev. B 90, 085402 (2014). 
[20] Can Wang, Qinglin Xia, Yaozhuang Nie, and Guanghua Guo, "Strain-induced gap transition and anisotropic dirac-like cones in monolayer and bilayer phosphorene," Journal of Applied Physics 117, 124302 (2015).

[21] Ruixiang Fei and Li Yang, "Strain-engineering the anisotropic electrical conductance of few-layer black phosphorus," Nano Letters 14, 2884-2889 (2014).

[22] Rafael Roldn, Andrs Castellanos-Gomez, Emmanuele Cappelluti, and Francisco Guinea, "Strain engineering in semiconducting two-dimensional crystals," Journal of Physics: Condensed Matter 27, 313201 (2015).

[23] Sandhya Chintalapati, Lei Shen, Qihua Xiong, and Yuan Ping Feng, "Magnetism in phosphorene: Interplay between vacancy and strain," Applied Physics Letters 107, 072401 (2015).

[24] Feng Hao and Xi Chen, "First-principles study of the defected phosphorene under tensile strain," Journal of Applied Physics 120, 165104 (2016).

[25] Jie Ren, Chunxiao Zhang, Jin Li, Zhixin Guo, Huaping Xiao, and Jianxin Zhong, "Strain engineering of magnetic state in vacancy-doped phosphorene," Physics Letters A 380, 3270-3277 (2016).

[26] Wei Hu and Jinlong Yang, "Defects in phosphorene," The Journal of Physical Chemistry C 119, 20474-20480 (2015).

[27] Ting Hu and Jinming Dong, "Geometric and electronic structures of mono- and di-vacancies in phosphorene," Nanotechnology 26, 065705 (2015).

[28] Pooja Srivastava, K. P. S. S. Hembram, Hiroshi Mizuseki, Kwang-Ryeol Lee, Sang Soo Han, and Seungchul Kim, "Tuning the electronic and magnetic properties of phosphorene by vacancies and adatoms," The Journal of Physical Chemistry C 119, 6530-6538 (2015).

[29] Yiren Wang, Anh Pham, Sean Li, and Jiabao Yi, "Electronic and magnetic properties of transition-metal-doped monolayer black phosphorus by defect engineering," The Journal of Physical Chemistry C 120, 9773-9779 (2016).
[30] W. Kohn and L. J. Sham, "Self-consistent equations including exchange and correlation effects," Phys. Rev. 140, A1133-A1138 (1965).

[31] Paolo Giannozzi, Stefano Baroni, Nicola Bonini, Matteo Calandra, Roberto Car, Carlo Cavazzoni, Davide Ceresoli, Guido L Chiarotti, Matteo Cococcioni, Ismaila Dabo, Andrea Dal Corso, Stefano de Gironcoli, Stefano Fabris, Guido Fratesi, Ralph Gebauer, Uwe Gerstmann, Christos Gougoussis, Anton Kokalj, Michele Lazzeri, Layla Martin-Samos, Nicola Marzari, Francesco Mauri, Riccardo Mazzarello, Stefano Paolini, Alfredo Pasquarello, Lorenzo Paulatto, Carlo Sbraccia, Sandro Scandolo, Gabriele Sclauzero, Ari P Seitsonen, Alexander Smogunov, Paolo Umari, and Renata M Wentzcovitch, "Quantum espresso: a modular and open-source software project for quantum simulations of materials," Journal of Physics: Condensed Matter 21, 395502 (2009).

[32] John P. Perdew, Kieron Burke, and Matthias Ernzerhof, "Generalized gradient approximation made simple," Phys. Rev. Lett. 77, 3865-3868 (1996).

[33] Andrew M. Rappe, Karin M. Rabe, Efthimios Kaxiras, and J. D. Joannopoulos, "Optimized pseudopotentials," Phys. Rev. B 41, 1227-1230 (1990).

[34] A. S. Rodin, A. Carvalho, and A. H. Castro Neto, "Strain-induced gap modification in black phosphorus," Phys. Rev. Lett. 112, 176801 (2014).

[35] Alexandra Carvalho, Min Wang, Xi Zhu, Aleksandr S. Rodin, Haibin Su, and Antonio H. Castro Neto, "Phosphorene: from theory to applications," Nat. Rev. 1, 16061 (2016).

[36] Hendrik J. Monkhorst and James D. Pack, "Special points for brillouin-zone integrations," Phys. Rev. B 13, 5188-5192 (1976).

[37] Liangzhi Kou, Yandong Ma, Sean C. Smith, and Changfeng Chen, "Anisotropic ripple deformation in phosphorene," The Journal of Physical Chemistry Letters 6, 1509-1513 (2015). 